\title{
ÍNDICE AUTORREFERIDO PELA EOUIPE DE ORTOPEDIA SOBRE A PREVENÇÃO DE INFECÇÃO DO SÍTIO CIRÚRGICO
}

Objetivo: avaliar o índice autorreferido pela equipe de cirurgia ortopédica sobre as recomendações para prevenção de infecção do sítio cirúrgico. Método: Estudo descritivo realizado em dois hospitais situados em Belo Horizonte, Minas Gerais. Os dados foram analisados no programa Stata,14 por estatística descritiva. Resultados: Participaram 133 profissionais: 22,5\% ortopedistas, 7,5\% enfermeiros e 69,9\% técnicos de enfermagem. Observou-se predominância de mulheres (61,3\%), tempo de trabalho > 8 anos (58,6\%); 53,3\% dos médicos e $84,4 \%$ da equipe de enfermagem reportaram conhecer as medidas de prevenção. As medidas com menor índice autorreferido foram: descontaminação nasal, referida por 10,7\% dos médicos; banho pré-operatório 6,6\% dos médicos e 7,8\% da Enfermagem. Conclusão: Medidas consideradas padrão-ouro pelas recomendações apresentaram índices autorreferidos abaixo de 50,0\% em ambas categorias profissionais, fato que pode relacionar-se à tendência de considerar medidas mais conhecidas ou realizadas na prática.

Descritores: Segurança do paciente; Prevenção e Controle; Infecção da Ferida Cirúrgica; Equipe de Assistência ao paciente.

\section{ORTHOPEDIC SURGERY TEAM SELF-REFERENCED INDEX ON THE PREVENTION OF SURGICAL SITE INFECTION}

Objective: The objective was to evaluate the self-reported index by the orthopedic surgery team on international and national recommendations and guidelines for the prevention of surgical site infection. Method: This was a descriptive study performed on Surgical Centers from two large hospitals located in the city of Belo Horizonte, Minas Gerais. Data were analyzed in the program Stata, 14 through descriptive analysis. Results: A total of 133 professionals were eligible, $22.5 \%$ were orthopedic surgeons, $7.5 \%$ nurses and $69.9 \%$ nursing technicians. It was observed a predominance of women, $61.3 \%$; with work time> 8 years, $58.6 \%$; $53.3 \%$ of the physicians and $84.4 \%$ of the nursing team reported knowing as preventive measures. The lowest self-reported index was highlighted in measures such as indication of nasal decontamination in patients with MMR, 10.7\% by the medical staff; indication of preoperative bath, $6.6 \%$ by physicians and $7.8 \%$ by nursing staff. Conclusion: Measures considered gold standard by specific guidelines presented self-reported indexes below $50.0 \%$ for two professional categories. The which may be related to the tendency to consider measures that they know best practice.

Descriptors: Patient safety; Prevention and Control; Surgical wound infection; Patient Care Team.

\section{ÍNDICE AUTORREFERIDO POR EL EQUIPO DE ORTOPEDIA SOBRE LA PREVENCIÓN DE INFECCIÓN DEL SITIO QUIRÚRGICO}

Objetivo: evaluar el índice autorreferido por el equipo de cirugía ortopédica sobre las recomendaciones para prevención de infección del sitio quirúrgico. Método: Estudio descriptivo realizado en dos hospitales situados en Belo Horizonte, Minas Gerais. Los datos fueron analizados en el programa Stata, 14 por estadística descriptiva. Resultados: Participaron 133 profesionales: 22,5\% ortopedistas, 7,5\% enfermeros y 69,9\% técnicos de enfermería. Se observó predominancia de mujeres, $61,3 \%$; tiempo de trabajo> 8 años, 58,6\%; 53,3\% de los médicos y $84,4 \%$ del equipo de enfermería reportaron conocer las medidas de prevención. Las medidas con menor índice autorreferido fueron: descontaminación nasal, referida por el 10,7\% de los médicos; baño preoperatorio $6,6 \%$ de los médicos y 7,8\% de la enfermería. Conclusión: Medidas consideradas patrón-oro por las recomendaciones presentaron índices autorreferidos por debajo del 50,0\% en ambas categorías profesionales. Hecho que puede relacionarse con la tendencia a considerar medidas más conocidas o realizadas en la práctica.

Descriptores: Seguridad del paciente; Prevención y Control; Infección de la herida quirúrgica; Equipo de asistencia al paciente.

'Universidade Federal de Minas Gerais

Autor Correspondente: Taysa de Fátima Garcia - Email: taysafati@hotmail.com

Recebido: 14/01/2019 - Aceito: 21/11/2019 


\section{INTRODUÇÃO}

As Infecções de Sítio Cirúrgico (ISC) são consideradas uma das mais importantes dentre as Infecções Relacionadas à Assistência a Saúde (IRAS) e constituem as complicações mais comuns decorrentes do ato cirúrgico, apresentando ocorrência entre 3\% a 20\% no pós-operatório(1) apresentando impacto direto no tempo de permanência hospitalar, custos com tratamentos diagnósticos e terapêuticos, uso prolongado de antibióticos e a reabordagem cirúrgica, além de potencializar os riscos de morbimortalidade. ${ }^{(2-3)}$

Atualmente, as ISC representam a principal causa de infecção entre os pacientes hospitalizados nos serviços de saúde dos Estados Unidos. ${ }^{(4)}$ No Brasil, embora não hajam dados sistematizados acerca da ocorrência das ISC, estas são apontadas em terceiro lugar entre as IRAS, apresentando taxas entre $14 \%$ a $16 \%$ aproximadamente. ${ }^{(1-2)}$

No contexto epidemiológico das ISC destacam-se as ocorridas em cirurgias ortopédicas dependentes do uso de implantes. ${ }^{(2.5)}$ A média das taxas de infecção cirúrgica após avaliação de 21.770 pacientes submetidos a cirurgia de quadril e joelho, foi de $1,7 \%$ a $2,8 \%$ em pacientes submetidos a artroplastias de quadril. ${ }^{6}$ No período de 2012 a 2014 as taxas de infecção avaliadas em 312 cirurgias de artroplastia de quadril e joelho e foi de 1,7\% e 1,2\% para ambas as cirurgias, respectivamente. (7)

É importante ressaltar, que o aumento da longevidade populacional predispõe o desenvolvimento de doenças agudas ou crônicas como artrites e desgastes ósseos, levando à necessidade de tratamentos cirúrgicos, sobretudo os relacionados à ortopedia ${ }^{(8)}$, o que pode repercutir diretamente na ocorrência da ISC( ${ }^{(9)}$.

No entanto, estima-se que as ISC podem ser evitadas em até $60 \%$ dos casos quando medidas para sua prevenção são aplicadas conforme as recomendações das diretrizes específicas. ${ }^{1}$ Destaca-se que a prevenção é multifatorial e depende da adesão multiprofissional ao que é preconizado e reconhecido por meio de evidências cientificas para boas práticas no perioperatório, enfatizando principalmente o transoperatório, que consiste no momento mais crítico da assistência ao paciente cirúrgico. ${ }^{(10)}$

Todavia, o conhecimento da equipe de cirurgia, especialmente médicos e equipe de enfermagem, pode não alcançar as recomendações dos guidelines e diretrizes específicas comprometendo seu cumprimento em cada momento da assistência ao paciente. Embora, muitas vezes a equipe cirúrgica afirme conhecer os fatores de risco para o desenvolvimento da infecção cirúrgica, as ações para sua prevenção ainda não acontecem na mesma proporção, devido a fatores como a subjetividade na interpretação das recomendações, ou devido a não aplicação e adesão efetiva a essas medidas, resultando no seu descumprimento, afetando diretamente no controle e prevenção das ISC.(11)

Nesse sentido, este estudo teve como objetivo avaliar o indice autorreferido pela equipe de cirurgia ortopédica sobre as recomendações e diretrizes internacionais e nacionais para a prevenção de infecção do sítio cirúrgico.

\section{MÉTODO}

Tipo de Estudo: Tratou-se de um estudo transversal com delineamento descritivo, realizado em Centros Cirúrgicos de dois hospitais de grande porte situados no município de Belo Horizonte, Minas Gerais.

Periodo: $\mathrm{O}$ estudo foi realizado entre os meses de junho a outubro de 2016

População e amostra: A população do estudo foi composta pelos profissionais que compunham a equipe de cirurgia ortopédica, que, neste estudo, considerou-se cirurgiões ortopedistas (preceptores e residentes), técnicos de enfermagem e enfermeiros, trabalhadores dos referentes centros cirúrgicos. Para composição, considerou-se amostra de conveniência, e incluiu cirurgiões preceptores, médicos residentes a partir do primeiro ano de residência, enfermeiros e técnicos de enfermagem do centro cirúrgico. Foram excluídos os profissionais sob licença médica, férias ou folga durante a realização da pesquisa, e aqueles que após a terceira tentativa de abordagem, não foram encontrados ou não responderam ao questionário.

Devido ao baixo número amostral de profissionais participantes, optou-se por agrupar preceptores e residentes na categoria "equipe médica", e enfermeiros e técnicos de enfermagem na categoria "equipe de enfermagem" partindo do princípio de que, a execução de funções e o conhecimento acerca da segurança do paciente no que diz respeito à prevenção de ISC, das duas categorias, sejam semelhantes.

Coleta de Dados: Foram realizadas visitas diárias aos centros cirúrgicos, e os profissionais, após aceitarem o convite à pesquisa, e assinar o Termo de Consentimento Livre e Esclarecido (TCLE), foram conduzidos a uma entrevista face a face, com um questionário estruturado, acerca de suas caracteristicas sociodemográficas e medidas de prevenção da infecção do sítio cirúrgico, de acordo com as diretrizes especificas.

As entrevistas ocorreram respeitando a dinâmica de trabalho e a disponibilidade dos profissionais, visando não interferir no fluxo e atendimento dos setores, e os respondentes receberam um caderno contendo as questões abertas, sem opções de resposta, o que permitiu que elaborassem as próprias respostas fundamentadas no seu domínio e conhecimento.

Análise dos dados: Os dados obtidos foram analisados no programa Stata, versão 14 por meio de estatística descritiva, medidas de tendência central e de dispersão. 
Procedimentos éticos: $O$ estudo respeitou as exigências formais contidas nas normas nacionais e internacionais regulamentadoras de pesquisas envolvendo seres humanos, com apresentação do TCLE a cada participante, e foi aprovado pelo Comitê de Ética e Pesquisa CAAE: 53645715.0.0000.5149.

\section{RESULTADOS}

Participaram deste estudo um total de 133 profissionais, sendo $30(22,5 \%)$ cirurgiões ortopedistas, 10 (7,5\%) enfermeiros e 93 (69,9\%) técnicos de enfermagem, constituindo $71,5 \%$ da amostra total dos dois hospitais elegiveis para o estudo.

No que diz respeito às características sociodemográficas, observou-se predomínio de mulheres 61,3\%; 55,6\% dos participantes eram casados, possuíam tempo de atuação profissional entre 1 a 36 anos, com mediana de 8 anos e desvio-padrão de 9,3. A idade variou entre 22 a 68 anos, com mediana de 35 anos e desvio-padrão de 9,7 (Tabela 1).

Tabela 1 - Características sociodemográficas dos profissionais participantes do estudo. Belo Horizonte, MG, Brasil, 2016. (n=133)

\begin{tabular}{lc}
\multicolumn{1}{c}{ Variáveis } & $\mathrm{n}(\%)$ \\
\hline Sexo & \\
Feminino & $82(61,6)$ \\
Masculino & $51(38,3)$ \\
\hline Idade (anos) & \\
\hline 35 & $62(46,6)$ \\
\hline 35 & $71(53,4)$ \\
\hline Formação Profissional & \\
\hline Médico & $30(22,5)$ \\
Enfermeiro & $10(7,5)$ \\
\hline Técnico de Enfermagem & $93(69,9)$
\end{tabular}

\section{Estado civil}

Solteiro

Casado

Divorciado

$$
\begin{aligned}
& \text { Tempo de atuação profissional (anos) } \\
& <8
\end{aligned}
$$

Tempo na instituição (anos)

$<3$

$>3$
O turno de trabalho predominante foi manhã, (36,8\%), seguido da tarde (33,8\%), 7,5\% à noite e $21,8 \%$ eram plantonistas. Sobre trabalhar em outra instituição, $77,4 \%$ não trabalhavam e $22,5 \%$ possuiam outro emprego na área, destes, $66,6 \%$ em mais uma instituição, 10,0\% em duas, 16,6\% em três e 6,7\% acima de três. Dos profissionais entrevistados que possuiam formação superior, 40,0\% dos médicos e 90,0 dos enfermeiros declararam ter alguma especialização, destes, $16,6 \%$ dos médicos possuíam mestrado, $8,3 \%$ doutorado, e $83,3 \%$ possuíam alguma especialização latu sensu, nenhum enfermeiro possuía mestrado ou doutorado

No tocante às medidas de prevenção da infecção do sítio cirúrgico, observou-se que $53,3 \%$ dos médicos e $84,4 \%$ da equipe de enfermagem reportaram conhecer as medidas e $66,6 \%$ e $56,3 \%$ das respectivas equipes relataram ter recebido algum treinamento sobre o assunto nos anos de 2015 ou 2016.

Quando questionado sobre seguir alguma diretriz ou guideline para prevenir a infecção cirúrgica $63,3 \%$ dos médicos e $60,2 \%$ da equipe de enfermagem afirmou adotar alguma recomendação. No entanto $79,1 \%$ não souberam responder qual diretriz era adotada, destes, $17,2 \%$ dos médicos e $82,8 \%$ da equipe de enfermagem. Dentre as diretrizes mencionadas 6,1\% relataram as da Agência Nacional de Vigilância Sanitária, $3,7 \%$ o Center for Disease Control and Prevention, 2,4\% a Organização Mundial da Saúde e 8,7\% referiram as recomendações/protocolos institucionais.

Após a categorização das questões elegiveis para avaliar o índice autorreferido pelos profissionais, no pré-operatório, transoperatório e pós-operatório, em consonância com os guidelines específicos, obtiveram-se os valores gerais de média do índice autorreferido por categoria profissional, representado no Gráfico 1 .

GRÁFICO 1 - Média do índice autorreferido por categoria profissional acerca das medidas de prevenção da infecção do sítio cirúrgico no pré-operatório, transoperatório e pósoperatório. Belo Horizonte, MG, Brasil, 2016. (n=133).

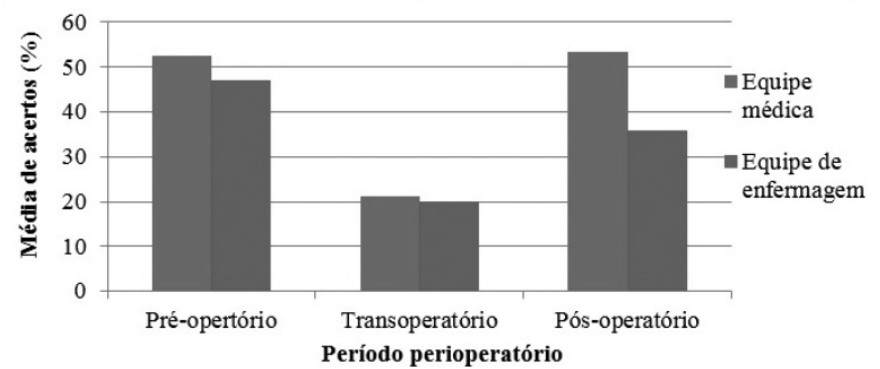

É possivel observar, que embora os profissionais afirmem conhecer as medidas de prevenção da infecção do sítio cirúrgico, o índice autorreferido para cada período do perioperatório estiveram aquém do esperado, enfatizando-se que o 
esperado acerca de conhecer as recomendações seja $100 \%$. A equipe médica apresentou um índice de $52,5 \%$ no pré-operatório; $21,1 \%$ no transoperatório $53,3 \%$ no pós-operatório. A equipe de enfermagem obteve média de $46,9 \%$ no pré-operatório, $19,9 \%$ no transoperatório e $35,9 \%$ no pós-operatório.

As medidas que representaram índice autorreferido menor que 50\% pela equipe cirúrgica, estão representados no Gráfico 2. E as principais do pré-operatório foram: a realização da tricotomia em uma antessala e na sala de cirurgia, 0,9\% da equipe de enfermagem; a indicação da descontaminação nasal em pacientes portadores de microrganismos multirresistentes foi mencionada apenas por médicos, apresentando indice autorreferido de 10,7\%, bem como o protocolo de descontaminação nasal referido por $26,6 \%$ desta categoria.

GRÁFICO 2 - Medidas com menor índice autorreferido acerca das medidas de prevenção da infecção do sítio cirúrgico, por categoria profissional, no pré-operatório, transoperatório e pós-operatório, (n=133), Belo Horizonte, 2016.

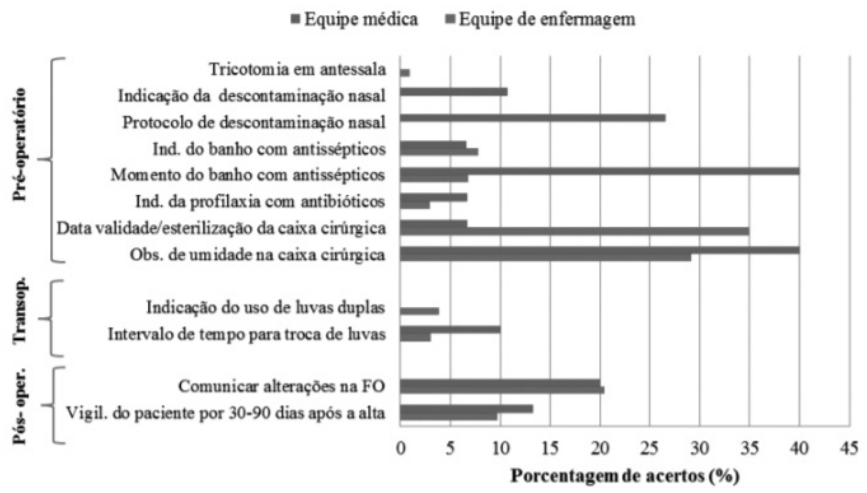

No tocante ao banho pré-operatório 6,6\% dos médicos e $7,8 \%$ da equipe de enfermagem referiram sua indicação em cirurgias de grande porte com inserção de implantes; 40,0\% e $6,8 \%$ das respectivas equipes mencionaram que este banho deve ocorrer na noite que antecede a na manhã da cirurgia. Em relação ao uso profilático de antibióticos somente 6,7\% da equipe médica e 2,9\% da enfermagem afirmaram que esta é uma medida recomendada para cirurgias limpas com inserção de implantes. Acerca das condições de esterilização do instrumental cirúrgico, 40,0\% dos cirurgiões e $29,1 \%$ da enfermagem citaram que a presença de umidade na caixa cirúrgica deve ser observada antes de sua abertura, e, 6,7\% e $34,9 \%$ das respectivas equipes mencionaram a verificação da data de esterilização e validade do material cirúrgico.

No transoperatório, as medidas menos autorreferidas foram a indicação do uso de luvas duplas em cirurgias de grande porte com inserção de implantes, mencionada apenas pela equipe de enfermagem, 3,9\%; e o intervalo de tempo em que as luvas devem ser trocadas (a cada 90 minutos) citada por 10,0\% dos médicos e 3,0\% da Enfermagem.
Dentre as medidas do pós-operatório que apresentaram menor índice autorreferido pelos profissionais, destacaram-se: orientar ao paciente a comunicar qualquer alteração na ferida operatória, 20,0\% dos médicos e 20,4\% da enfermagem; e, realizar a vigilância do paciente após a alta hospitalar no período de 30 a 90 dias, que foi mencionada por 13,3\% e $9,7 \%$ respectivamente.

No entanto, houveram medidas em que os profissionais referiram a alternativa correta em uma porcentagem maior que $50 \%$. Dentre elas, se destacaram o descarte das lâminas do tricotomizador a cada uso, reportada por $80,0 \%$ dos médicos e 74,1\% da equipe de enfermagem; a administração de antibióticos profiláticos de 30 a 60 minutos antes da cirurgia, mencionada por $100,0 \%$ dos médicos e $82,5 \%$ da enfermagem; e o tempo dispendido para o preparo cirúrgico das mãos, referido por $88,8 \%$ e $65,0 \%$ das respectivas equipes.

A necessidade de troca das luvas antes da colocação de implantes, foi referida por $60,0 \%$ da equipe médica e quando houverem perfurações/abrasões citada por 52,4\% da enfermagem. E manter o curativo por 24 a 48 horas após a cirurgia foi mencionada por $93,3 \%$ dos médicos e $62,1 \%$ da equipe de enfermagem.

\section{DISCUSSÃO}

Neste estudo a maioria dos profissionais foram mulheres (61,3\%), especialmente devido ao grande número de profissionais correspondente à equipe de enfermagem, corroborando resultados outros achados que reafirmam o predomínio de trabalhadores do sexo feminino em profissões como enfermagem. ${ }^{(12)}$ Com relação à faixa etária, a maioria dos participantes possuíam idade $>35$ anos $(53,4 \%)$ e tempo de atuação profissional de $>8$ anos (58,6\%), em consonância com outro estudo que avalia o perfil de trabalhadores de saúde em que os achados foram semelhantes à deste, com média de idade de 32,7 anos (mínimo 21 e máximo 54 anos) e tempo de atuação entre cinco a dez anos. ${ }^{(13)}$

Com relação ao índice autorreferido pelos profissionais, medidas como a realização da tricotomia em antessala, fora da sala de cirurgia foi referido por apenas 0,9\% da equipe de enfermagem, $100,0 \%$ da equipe médica e 99,0\% da equipe de enfermagem relataram que esta deve ser realizada dentro da sala de cirurgia antes do preparo da pele do paciente.

Dentre as recomendações dos guidelines têm-se que, a remoção dos pelos deve ocorrer apenas se houver absoluta necessidade, e deve ocorrer em uma antessala da sala de cirurgia, mais próximo possível do horário do procedimento com uso de tricotomizadores elétricos, de lâminas descartáveis, que façam tonsura dos pêlos, a fim de prevenir pequenos abrasões ou lacerações na pele do paciente que podem predispor abertura para $\mathrm{O}$ alojamento de bactérias e, portanto à contaminação aumentando o risco de ocorrência de infecção. . $^{(1,3,5,10,14)}$ 
No tocante à descontaminação nasal em pacientes portadores de microrganismos multirresistentes, ao serem questionados sobre a sua indicação apenas 10,7\% da equipe médica referiu a cirurgias de grande porte com inserção de implantes, conforme recomendado pelos guidelines. Cerca de 42,8\% médicos e 98,0\% enfermagem desconheciam a indicação. De acordo com as recomendações específicas, pacientes submetidos a cirurgias cardiotorácicas ou ortopédicas, sabidamente colonizados por estes microrganismos devem ser tratados por cinco dias, antes da cirurgia, com aplicação de mupirocina intranasal (2,0\%) a cada 12 horas, associado ou não com o banho com clorexidina. ${ }^{(1,3,10,15)}$

Sobre a indicação do banho pré-operatório apenas 6,6\% dos médicos e $7,8 \%$ da equipe de enfermagem citaram sua indicação correta e somente $40,0 \%$ e 6,8\% respectivamente, mencionaram que este banho deve ocorrer na noite que antecede a na manhã da cirurgia. Embora haja controvérsias sobre o uso de antissépticos no banho pré-operatório, no tocante à redução da infecção do sítio cirúrgico, a recomendação é que se realize o banho com antisséptico ${ }^{(16)}$ dois banhos com clorexidina antes da cirurgia, um na noite anterior e outro no dia da mesma. ${ }^{(3)}$ No entanto, a atualização das diretrizes brasileiras, recomendam um banho na noite anterior ou na manhã da cirurgia.(1)

O enluvamento duplo no transoperatório em cirurgias de grande porte com inserção de implantes, bem como seu intervalo de troca estão entre as recomendações mais importantes para prevenção da infecção em cirurgias ortopédicas dependentes de implantes, ${ }^{(15)}$ sobretudo devido ao alto risco de perfurações ou abrasões durante o procedimento cirúrgico, sejam decorrentes de defeitos da própria fabricação, ou do próprio ato cirúrgico, podendo consequentemente contaminar a incisão com microrganismos presentes nas mãos do cirurgião, sem que este perceba. ${ }^{(17-18)}$

O rompimento da integridade de luvas durante a realização de procedimentos cirúrgicos foi identificada em 11,1\% de 198 luvas avaliadas após cirurgias da clínica de ginecologia, destas que sofreram alguma danificação, cerca de 40,9\% ocorreram no tempo de uso entre 90 a 119 minutos de procedimento. ${ }^{(18)}$ Dado que corrobora com as recomendações sobre o intervalo de tempo recomendado para a troca de luvas, especialmente em cirurgias ortopédicas. ${ }^{(17)}$

Sobre o intervalo de tempo recomendado para a vigilância após a alta hospitalar, observou-se que uma pequena parcela dos profissionais referiram o período de 30 a 90 dias. De acordo com as novas diretrizes do Center for Diseases Control and Prevention estas cirurgias devem ser monitoradas, com potencial para ocorrência de infecção do sítio cirúrgico, por um período entre 30 a 90 dias a contar da data de realização do procedimento. ${ }^{(2,19)}$
Algumas medidas analisadas descritivamente sugerem que as práticas da equipe cirúrgica se distanciam do seu discurso. Práticas comuns à Enfermagem foram comumente citadas por médicos, como a observância da umidade do material estéril; bem como a referência pela equipe de enfermagem para o uso da luva dupla. Ressalta-se, a importância da discussão multidisciplinar acerca das práticas para prevenção da infecção cirúrgica ${ }^{(20)}$, e destaca-se, neste cenário, o papel essencial da liderança em enfermagem que deve estar intimamente relacionada com o cuidado, emergindo em ações que possam ser norteadoras de mudanças ${ }^{(21)}$.

Com base nos resultados encontrados, infere-se que os profissionais possuem uma tendência a considerar àquilo que conhecem e/ou desempenham com maior frequência em sua prática diária, dentro de sua categoria, e não baseado no seu conhecimento e conjunto de boas práticas definidas e aceitas por evidencias cientificas, mas muitas vezes fundamentadas na sua percepção e interpretação subjetiva das recomendações.

\section{Limitações do estudo}

A principal limitação deste estudo se referiu à dificuldade em acessar a equipe cirúrgica diante da indisponibilidade para responder aos questionários, devido a alta demanda de atendimentos nas instituições e tempo escasso. Outra limitação importante neste estudo pode ser atribuída aos imprevistos relacionados ao agendamento cirúrgico, uma vez que as entrevistas e, portanto, o alcance à clínica de interesse ocorria dentro do centro cirúrgico. Houveram ainda entrevistas que foram iniciadas mas precisaram ser reprogramadas para outros dias ou horários, e àquelas que foram iniciadas, porém, em virtude do fluxo de atividades dos profissionais, não foram finalizadas, situação que ocorreu com seis profissionais médicos e quatro da enfermagem, resultando em perda amostral. Dentre os fatores facilitadores destacou-se a receptividade encontrada nas instituições participantes da pesquisa, desde a anuência à participação individual de cada profissional.

\section{Contribuições para a prática}

Destaca-se o feedback às instituições participantes, após seu interesse em conhecer os resultados a fim de produzir e melhorar os indicadores de qualidade assistencial, reconhecendo a segurança cirúrgica como um desafio global a ser trabalhada pela equipe multiprofissional em busca de estratégias para melhoria do cuidado dentro da instituição por meio do conhecimento, medida que nem sempre é valorizada pela equipe.

Diante dos resultados encontrados, este estudo aponta a importância e necessidade de que auditorias periódicas sejam 
realizadas a fim de proporcionar indicadores para uma assistência cirúrgica segura, haja vista os índices autorreferidos aquém do esperado podem estar relacionados a não adoção de boas práticas no centro cirúrgico para prevenção da ISC e segurança do paciente. Portanto a realização de estudos voltados para a avaliação do conhecimento da equipe multiprofissional devem ser encorajados, principalmente aqueles multicêntricos, visando investigar, além da atualização profissional, o seu comportamento mediante às inúmeras diretrizes e recomendações específicas para a segurança do paciente.

\section{CONCLUSÃO}

Este estudo apontou fragilidades quanto ao índice autorreferido acerca de boas práticas para prevenir a infecção do sítio cirúrgico, conforme preconizado pelas diretrizes específicas. Medidas consideradas como padrão-ouro, como: local para realização da tricotomia, indicação e protocolo de descontaminação nasal em portadores de microrganismos multirresistentes, momento ideal para o banho com uso de antissépticos, indicação da profilaxia com antibióticos, observação das condições de esterilização do instrumental cirúrgico, indicação do uso de luvas duplas bem como seu intervalo de troca e vigilância do paciente após a alta hospitalar (por 30 a 90 dias), apresentaram índices autorreferidos abaixo de $50 \%$ por ambas categorias profissionais.

Nesse sentido, a discussão e treinamento multiprofissional acerca da prevenção é essencial, reforçando o impacto das ações, individuais e coletivas, no cuidado ao paciente cirúrgico maneira integralizada.

\section{PARTICIPAÇÕA DOS AUTORES}

Todos os autores contribuiram equitativamente na concepção do desenho do estudo, na análise e interpretação dos dados bem como na redação do artigo, desde a sua fase inicial à revisão crítica e revisão final.

\section{REFERÊNCIAS}

Agência Nacional de Vigilância Sanitária. Medidas de Prevenção de infecção relacionada à assistência à saúde. Brasilia: ANVISA. Brasil. [Internet]. 2017 [cited 2017 Jul 22]; 1-201. Available from: https://www20.anvisa.gov. br/segurancadopaciente/index.php/publicacoes/item/medidas-de-prevencao-de-infeccao-relacionada-aassistencia-a-saude-3

Agência Nacional de Vigilância Sanitária. Critérios diagnósticos de infecção relacionada à assistência à saúde. Brasilia: ANVISA. Brasil. [Internet]. 2017 [cited 2017 Jul 22]; 1-86. Available from: https://www20.anvisa.gov. br/segurancadopaciente/index.php/publicacoes/item/criterios-diagnosticos-das-infeccoes-relacionadas-aassistencia-a-saude

National Institute for Health and Clinical Excellence. Surgical site infection prevention and treatment of surgical site infection. NICE. [Internet]. 2008 [cited 2016 Jan 22]; 1-168. Available from: https://www.nice.org. uk/guidance/cg74/evidence/full-guideline-242005933

Magill SS, Edwards JR, Bamberg W, Beldavs ZG, Dumyati G, Kainer MA, et al. Multistate Point-Prevalence Survey of Health Care-Associated Infections. N Engl J Med Overseas [Internet]. 2014 [cited 2016 Set 22]; 370(13):1198-208. Available from: https://www.ncbi.nlm.nih.gov/pubmed/24670166

Berríos-Torres SI, Umscheid CA, Bratzler DW, Leas B, Stone EC, Kelz RR, et al; Healthcare Infection Control Practices Advisory Committee. Centers for Disease Control and Prevention guideline for the prevention of surgical site infection. JAMA Surg [Internet]. 2017 [cited 2017 Out 10]; 3:1-600. Available from: https://www. ncbi.nlm.nih.gov/pubmed/28467526

Kim JL, Park JH, Han SB, Cho IY, Jang KM. Allogeneic Blood Transfusion Is a Significant Risk Factor for Surgical-Site Infection Following Total Hip and Knee Arthroplasty: A Meta-Analysis. J Arthroplasty [Internet] 2017 [cited 2017 Jul 12]; 32:320-25. Available from: https://www.ncbi.nlm.nih.gov/pubmed/27682006

May ER, Bush K, Vickers D, Smith S. Use of a provincial surveillance system to characterize postoperative surgical site infections after primary hip and knee arthroplasty in Alberta, Canada. Am J Infec 
Control [Internet]. 2016 [cited 2017 Jan 25]; 44:1310-14. Available from: https://www.ncbi.nlm.nih.gov/ pubmed/27158089

Roger E, Guillaume V, Julien D, Nicolas R, Stéphane D, Stéphane B. Progression and projection for hip surgery in France, 2008-2070: Epidemiologic study with trend and projection analysis. Orthopaedics \& Traumatology: Surgery \& Research [Internet] 2019 [cited 2019 Nov 06]; 105:1227-35. Available from: https://www.ncbi.nlm.nih. gov/pubmed/31606338

Fu-Der W, Yu-Ping W, Cheng-Fong C, Hsin-Pai C. The incidence rate, trend and microbiological aetiology of prosthetic joint infection after total knee arthroplasty: A 13 years' experience from a tertiary medical center in Taiwan. Journal of Microbiology, Immunology and Infection. [Internet] 2018 [cited 2019 Nov 06]; 51:717e722030. Available from: https://www.ncbi.nlm.nih.gov/pubmed/30228089

World Health Organization. Global Guidelines for the Prevention of Surgical Site Infection, Geneva: WHO [Internet]. 2016 [cited 2017 Mar 10]; Available from: http://www.who.int/gpsc/global-guidelines-web.pdf

Filho GRM, Silva LFN, Ferracini AM, Bahr GL. Protocolo de Cirurgia Segura da OMS: O grau de conhecimento dos ortopedistas brasileiros. Rev Bras Ortop [Internet] 2013 [cited 2016 Mar 11]; 48(6):554-62. Available from: http://www.rbo.org.br/PDF/48-6-port/19-103.pdf

Cabral RA, Eggenberger T, Keller K, Gallison BS, Newman D. Use of a Surgical Safety Checklist to Improve Team Communication. AORN J [Internet] 2016 [cited 2016 Fev 19]; 104:206-16. Available from: https://www. ncbi.nlm.nih.gov/pubmed/27568533

Araujo MAN, Filho WDL, Alvarenga MRM, Oliveira RD, Souza JC, Vidmantas S. Perfil sociodemográfico dos enfermeiros da rede hospitalar. Rev enferm UFPE. [Internet] 2017 [cited 2019, nov 06]; 11:4716-25. Available from: https://pdfs.semanticscholar.org/efOb/50dOfOebelcabd903080f3b67fclf920b3e6.pdf

Charles JREE, Russell GK, Judith T, Maureen S, Gary SR, David L L. Perioperative hair removal in the 2lst century: Utilizing an innovative vacuum-assisted technology to safely expedite hair removal before surgery. American Journal of Infection Control [Internet] 2016 [cited 2019 Nov 06]; 44:1639-44. Available from: https:// www.ncbi.nlm.nih.gov/pubmed/27375061

National Health and Medical Research Council. Australian Comission on Safety and Quality in Healthcare. Australian guidelines for the prevention and control of infection in healthcare. NHMRC [Internet] 2010 [cited 2015 Set 10]; Available from: https://www.nhmrc.gov.au/health-topics/infection-prevention-and-control

Franco LMC, Cota GF, Pinto TS, Ercole FF. Preoperative bathing of the surgical site with chlorhexidine for infection prevention: Systematic review with meta-analysis. American Journal of Infection Control. [Internet] 2017 [cited 2019 nov 07]; 45:343-9. Available from: https://www.ncbi.nlm.nih.gov/pubmed/28109628

Gehrke T, Parvizi J. Proceedings of the International Consensus Meeting on Periprosthetic Joint Infection. Filadélfia: International Consensus Group [Internet] 2013 [cited 2015 Set 11]; Available from: https://www.ncbi. nlm.nih.gov/pubmed/24151261

Oliveira AC, Gama CS. Antissepsia cirúrgica e utilização de luvas cirúrgicas como potenciais fatores de risco para contaminação transoperatória. Esc Anna Nery [Internet] 2016 [cited 2017 Jan 18]; 20(2):370-77. Available from: http://www.scielo.br/pdf/ean/v20n2/1414-8145-ean-20-02-0370.pdf

Center for Diseases Control and Prevention. The National Healthcare Safety Network (NHSN). Patient Safety Component Manual. CDC [Internet]. 2019 [cited 2019 Nov 07]; 408p. Available from: https://www.cdc.gov/ nhsn/pdfs/pscmanual/pcsmanual_current.pdf

Barnes S. Infection Prevention: The Surgical Care Continuum. AORN J [Internet] 2015 [cited 2016 Jan 23]; 101(5):512-18. Available from: https://www.ncbi.nlm.nih.gov/pubmed/25946177

Pereira LA, Ramos AM, Schallenberger CD, Vaz MC, Barlem ELD. Liderança em enfermagem: abordagem ecossistêmica com impacto no cuidado. Enferm. Foco [Internet] 2018 [cited 2019 nov 07]; 9(3): 66-70. Available from: http://revista.cofen.gov.br/index.php/enfermagem/article/view/1351/463 